Revisión

\title{
Desarrollo y aportes desde una psicología positiva de la salud al abordaje de la salud / enfermedad
}

\author{
María Emma Reyes-Cuervo ab 1 \\ https://orcid.org/0000-0003-4902-5324 \\ Stefano Vinaccia Alpi ${ }^{\mathrm{c}}$ \\ https://orcid.org/0000-0001-5169-0871 \\ ${ }^{a}$ Escuela Colombiana de Rehabilitación, Bogotá, Colombia \\ ${ }^{\mathrm{b}}$ Universidad de Palermo, Buenos Aires, Argentina \\ ${ }^{\mathrm{c}}$ Universidad del Sinú, Montería, Colombia
}

Fecha de recepción: 02 de mayo de 2019

Fecha de aprobación: 15 de julio de 2019

\section{Resumen}

La APA reconoce 54 divisiones a manera de grupos de interés entre las que están la psicología de la salud y la psicología de la rehabilitación. Además, quien fuera presidente de esta asociación en 1998 impulsó la psicología positiva como perspectiva desde la necesidad de virar la atención hacia el funcionamiento psíquico óptimo y con ello a las fortalezas y virtudes humanas. Objetivo: explorar en la literatura científica sobre salud física los abordajes bajo una perspectiva de la psicología positiva. Método: búsqueda detallada en PsycArticles con los términos "positive psychology" AND "health" en AB (abstract). Se revisaron 16 artículos luego de seleccionar solo aquellos que abordaron condiciones de salud física particulares bajo una mirada desde la psicología positiva. Se realizó una exploración inicial de contenido y un análisis de los hallazgos y discusiones de los artículos. Resultados: Se presentan resultados para 11 enfermedades o deficiencias de salud objeto de aproximación desde la psicología positiva en los artículos revisados (cáncer, diabetes, enfermedad coronaria, enfermedad musculoesquelética, enfermedad cerebrovascular, lesión medular, obesidad, esclerosis múltiple, fibromialgia, abuso de sustancias y síntomas físicos) y las variables o categorías positivas que se consideraron en las investigaciones (optimismo, resiliencia, crecimiento postraumático, bienestar, satisfacción con la vida, afecto positivo, sentido, adherencia al tratamiento, calidad de vida y atención plena). Así mismo se reseñan las intervenciones positivas y las perspectivas futuras que se desprendieron de la revisión. Discusión: delimitar una psicología positiva de la salud requiere fortalecer la producción y la evidencia científica en esta materia. Se trata de un campo prometedor tanto en el desarrollo de estrategias de prevención y educación para la salud como de intervención en deficiencias de salud específicas, encaminado en todo caso, al bienestar y calidad de vida relacionado con la salud especialmente.

Palabras clave: Psicología; estado de salud; salud mental; optimismo; psicología médica; psicología positiva de la salud.

\footnotetext{
${ }^{1}$ Correo de contacto: mariaereyes@gmail.com
}

DOI: 10.30788/RevColReh.v18.n2.2019.412

Esta obra se encuentra bajo licencia internacional CC BY 4.0 Institución editora: Escuela Colombiana de Rehabilitación 


\title{
Development and contributions from a positive health psychology approach to the health / disease
}

\begin{abstract}
The APA recognizes 54 divisions as interest groups including the psychology of health and the psychology of rehabilitation. In addition, who was the president of this association in 1998 promoted positive psychology as a perspective from the need to turn attention to optimal psychic functioning and thus to human strengths and virtues. Objective: to explore in the scientific literature on physical health the approaches from a perspective of positive psychology. Method: A search in PsycArticles with the keywords "positive psychology" AND "health" in AB (abstract). 16 articles were reviewed after selecting only those that addressed to particular physical health conditions under a view from positive psychology. An initial content exploration and an analysis of the findings and discussions of the articles was conducted. Results: Results are presented for 11 diseases or health deficiencies subject to approximation from positive psychology in the articles (cancer, diabetes, cardiovascular disease, musculoskeletal disease, cerebrovascular disease, spinal cord injury, obesity, multiple sclerosis, fibromyalgia, substance abuse and physical symptoms) and the positive variables or categories that were considered in research (optimism, resilience, post-traumatic growth, well-being, satisfaction with life, positive affect, meaning, adherence to treatment, quality of life and mindfulness). It also outlines the positive interventions and future prospects that were detached from the review. Discussion: to delimit a positive health psychology requires strengthening the production and scientific evidence in this area. It is a promising field both in the development of health prevention and education strategies and intervention in specific deficiencies, aimed in any case at health-related quality of life and well-being.
\end{abstract}

Keywords: psychology; health status; mental health; optimism; psychology, medical; positive health psychology.

\section{Introducción}

La psicología positiva se entiende como el uso de la teoría psicológica, sus métodos y sus técnicas para comprender los aspectos positivos del comportamiento humano, de manera tal que dedica su empeño al estudio del funcionamiento psíquico óptimo (Alex Linley, Joseph, Harrington, \& Wood, 2006). Es una perspectiva orientada al conocimiento sobre los recursos psicológicos de las personas y su aporte en el bienestar subjetivo, la calidad de vida y la salud; en contraposición con el estudio de lo patógeno, propio del modelo biomédico que imperó en la primera mitad del siglo XX (Contreras \& Esguerra, 2006). Su surgimiento y desarrollo es atribuido a Martín Seligman, por ser él, quien en 1998 desde la presidencia de la American Psychological Asociation (APA), propuso un movimiento para tomar distancia prudente del abordaje sobre el daño y la debilidad, y conducir una porción de la psicología científica de vuelta al estudio de las fortalezas y las virtudes humanas (Seligman, 2003). Idea bien recibida en un contexto propicio cuando el modelo psicosocial en salud se encontraba fortalecido.

Debe decirse, que el estudio de categorías y variables directamente relacionadas con el bienestar ha sido siempre de interés de la psicología científica, de hecho, desde tiempo atrás asuntos como la creatividad, la autoestima, la ética o los valores fueron recogidos por la psicología humanística, o el afrontamiento, la autoeficacia, el control percibido o el vínculo afectivo fueron objeto de la psicología conductual. Pero fue Seligman quien, hace dos décadas, propició la integración de este conocimiento en un cuerpo teórico común 
de base empírica muy importante en el desarrollo de la psicología científica (Lupano Perugini \& Castro Solano, 2010), a la vez que posibilitó el impulso de un programa de investigación, que recupera los aspectos positivos del comportamiento humano en diferentes campos de aplicación, entre los que se cuentan el educativo, el laboral, el clínico y el de la salud (Contreras \& Esguerra, 2006).

Desde sus inicios, la psicología ha estado involucrada con la salud; no sorprende que varios de los primeros psicólogos se iniciaron en la medicina (es el caso de Helmholtz, James o Wundt) y los desarrollos sobre medicina psicosomática de los años 40 resultaron cruciales en la aproximación de los aspectos psicológicos a la salud física, particularmente en la reflexión sobre los servicios médicos; o los de la medicina conductual, que en los años 70 aproximaron los métodos y técnicas biomédicas y comportamentales de la psicología conductual, al ámbito de la salud y la enfermedad.

Tales antecedentes conformaron el caldo de cultivo propicio para el surgimiento y desarrollo de la psicología de la salud en los años 80, que se constituyó desde entones en un área propia de la psicología como disciplina (y ya no como campo interdisciplinar), reconocida como división de la APA (div. 38) y que en términos de Matarazzo (1980) se dedica a "la promoción y mantenimiento de la salud, la prevención y tratamiento de la enfermedad, la identificación de correlatos etiológicos y diagnósticos de la salud, de la enfermedad y de las disfunciones afines, y para el análisis y mejora del sistema sanitario, así como para la configuración de las políticas sanitarias" (p. 815). La psicología de la rehabilitación por su parte, como otra de las áreas de la psicología en contextos de salud y reconocida como una división de la APA (div. 22), cobra fuerza posteriormente al amparo de la Convención Internacional sobre los Derechos de las Personas con Discapacidad (CRPD por su sigla en inglés) en 2008. (Reyes-Cuervo \& Bejarano-Celis, 2013).

Específicamente en la psicología de la salud y la de la rehabilitación, la psicología positiva constituye una fuerza con importante influencia sobre la investigación y el desarrollo teórico. Múltiples constructos se han apuntalado bajo la sombrilla de la psicología positiva y se han incorporado a la comprensión de procesos salutogénicos relevantes para la prevención y la promoción de la salud, incluso cuando no existe dolencia o malestar (Schmidt, Raque-Bogdan, Piontkowski, \& Schaefer, 2011; Riveros \& Vinaccia-Alpi, 2017). Howell, Kern y Lyubomirsky (2007) revelaron mediante un meta-análisis que el bienestar tiene un efecto importante sobre la salud y que esto ocurre aún en ausencia de enfermedad. Resiliencia, crecimiento postraumático, espiritualidad, emociones positivas, calidad de vida (Ehde, 2010; Park, 2015) así como el florecimiento o la salud psicológica (Thege et al., 2015) hacen parte del abanico de los constructos más importantes de la agenda investigativa de la psicología que se ha interesado en comprender los procesos de salud; tanto en contextos de enfermedad crónica tales como cáncer, diabetes, VIH, enfermedad pulmonar entre otras (Vinaccia \& Quiceno, 2012) como en contextos de la rehabilitación frente a la discapacidad, como lesión medular, amputación de miembros, enfermedad cerebrovascular, trauma cráneo-encefálico, dolor crónico y esclerosis múltiple, entre otros (Frank, Rosenthal, \& Caplan, 2010).

El propósito de esta revisión consiste en presentar los aportes de la psicología de la salud, incluida la psicología de la rehabilitación, que contempla como perspectiva la psicología positiva, tal exploración estuvo restringida a PsyArticles, una base que permite acceder al texto completo de los artículos de revistas publicadas por la American Psycological Asociation, la APA Educational Publishing Foundation, la Canadian Psychological Association y Hogrefe \& Huber. Esta base de datos incluye todo el material de las revistas impresas y recupera en la mayoría de los títulos el volumen 1, número 1. 
Se realizó una exploración previa de artículos disponibles en PsyArticles, incluyendo la palabra clave "health psychology" dentro del abstract, que arrojó el primer registro en la década de los 30, mientras que "rehabilitation psychology" presenta solo dos registros antes de 1940 y solo reaparece como categoría consolidada en la década de los 80. La psicología positiva en cambio, en congruencia con el protagonismo otorgado por su mentor en la APA, el Dr. Seligman, tiene apenas un par de registros en la década de los 90, apareciendo como categoría consolidada luego del año 2000. En síntesis, bajo esta exploración preliminar de los abstract de artículos disponibles en PsycArticles, el panorama de publicaciones muestra que mientras que en psicología de la salud son 350 registros, en psicología positiva son 108 y para psicología de la rehabilitación apenas 92. La figura 1 muestra la distribución de artículos por décadas para cada una de las tres categorías exploradas previa a la revisión documental que tuvo como propósito explorar en la literatura científica sobre salud física los abordajes bajo una perspectiva de la psicología positiva.

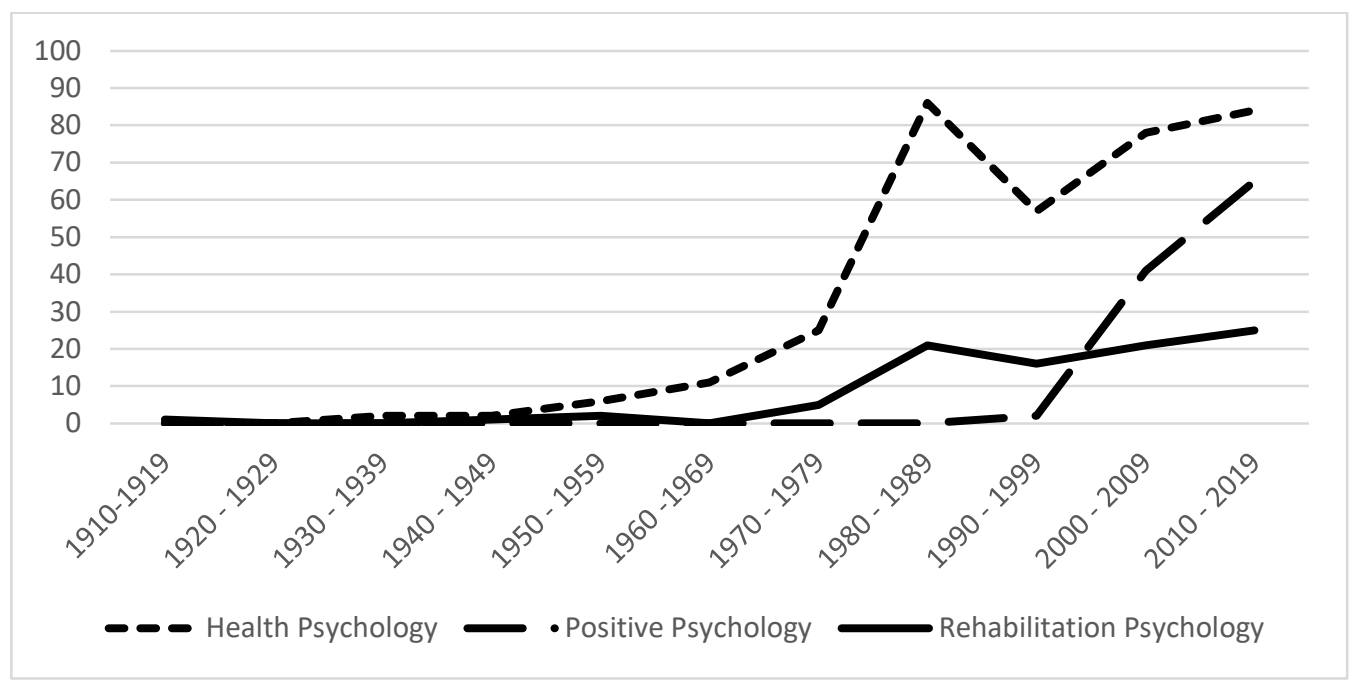

Figura 1. Número de artículos con referencia en el resumen a tres campos de la psicología por décadas desde 1910 hasta la actualidad

\section{Método}

Se realizó una búsqueda detallada en PsycArticles mediante el uso de términos como psicología positiva y salud: "positive psychology" AND "health" en AB (abstract) que arrojó un total de 168 registros entre 1946 y 2019 (con corte el 28 de febrero de 2019), las revistas con mayor número de artículos que incorporan esta combinación son la American Psychologist (22) y la Health Psychology (21), la mayor concentración de artículos se encuentra entre 2000 y 2019 con un total de 138 artículos, 17 más se encuentran en la década de los 90 y los restantes distribuidos unitariamente en años anteriores a 1989. De entre estos se seleccionaron, tras la lectura de los abstract, solo aquellos que incorporaron el abordaje de condiciones o enfermedades físicas, descartando tanto modelos teóricos o programas de intervención genéricos desde la psicología positiva como aquellos que se limitaron a una aproximación exclusiva sobre salud mental (en general o en particular sobre depresión, ansiedad, trauma o poblaciones particulares, entre otros). Un total de 16 artículos fueron incorporados a la presente revisión. Los documentos fueron sometidos a un análisis mediante exploración de contenido y en respuesta a las cuestiones de interés a propósito de una psicología positiva de la salud, a saber: ¿cuáles son las condiciones de salud física y las 
variables estudiadas bajo una perspectiva declarada de la psicología positiva?, y ¿Qué desarrollos en cuanto a intervención se han presentado y cuáles son las perspectivas futuras que de ellas se desprenden?

\section{Resultados}

La figura 2 muestra una exploración inicial del contenido mediante las palabras más frecuentes en los artículos revisados, que representa un primer esbozo de lo que le atañe a la salud positiva o psicología positiva de la salud en la literatura científica de la APA. La investigación psicológica revisada recoge estudios con mención a aspectos que remiten a variables observadas por la psicología positiva como son gratitud, afrontamiento, significado, atención plena, emoción, afecto, regulación o crecimiento. Son menos frecuentes las palabras con referencia directa a condiciones de salud particulares, deficiencias, enfermedades o patologías; tímidamente se registran los términos: físico, síntomas y enfermedad cerebrovascular junto con las referencias a depresión y ansiedad. Un desglose más profundo del contenido se presenta en lo que sigue.

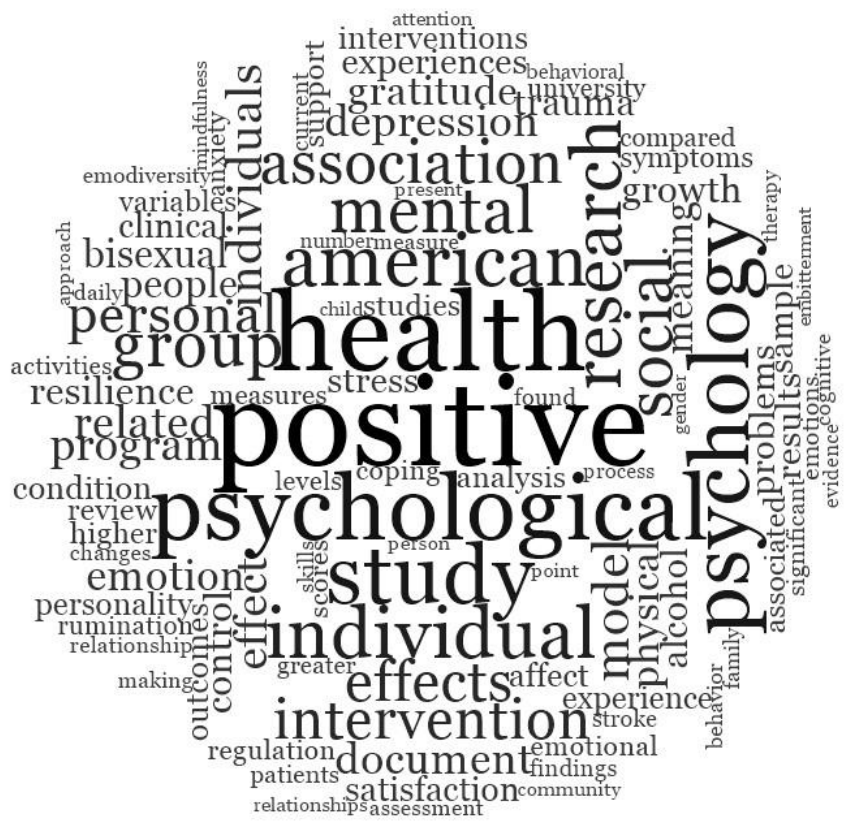

Figura 2. Palabras más frecuentes en el contenido de los artículos incorporados en la revisión.

\section{Condiciones de salud física y aspectos positivos estudiados}

El abordaje de la enfermedad física desde una perspectiva positiva ha supuesto la consideración de la salud positiva en diferentes escenarios tanto de prevención como de intervención. Patologías como cáncer (Gan, Zheng, \& Wang, 2018; Casellas-Grau, Font, \& Vives, 2014; Miller, Sherman, \& Christensen, 2010; Coyne et al., 2010) , diabetes (Celano et al., 2019; Bergner et al., 2018) y enfermedad cardiovascular (Labarthe et al., 2016; Burg, Wolf, \& Michalak, 2012) son las que se repiten con mayor frecuencia en los estudios reportados bajo la mirada de la psicología positiva. La figura 3 resume las temáticas centrales en lo concerniente a enfermedad física que abordan los artículos revisados.

Los autores refieren que, en diferentes condiciones de deficiencia en salud, las personas se perciben de cara a un gran conflicto ante el cual se sienten superadas por las circunstancias y con sentimientos de 
miedo, tristeza, rabia e incluso incertidumbre (Gan et al., 2018). De acuerdo con Nierenberg et al. (2016) cuando las personas se enfrentan a una discapacidad o enfermedad crónica, pueden llegar a niveles premórbidos de su funcionamiento psicológico, incluso desarrollando desórdenes especialmente en el estado de ánimo como depresión, ansiedad e incluso estrés postraumático.

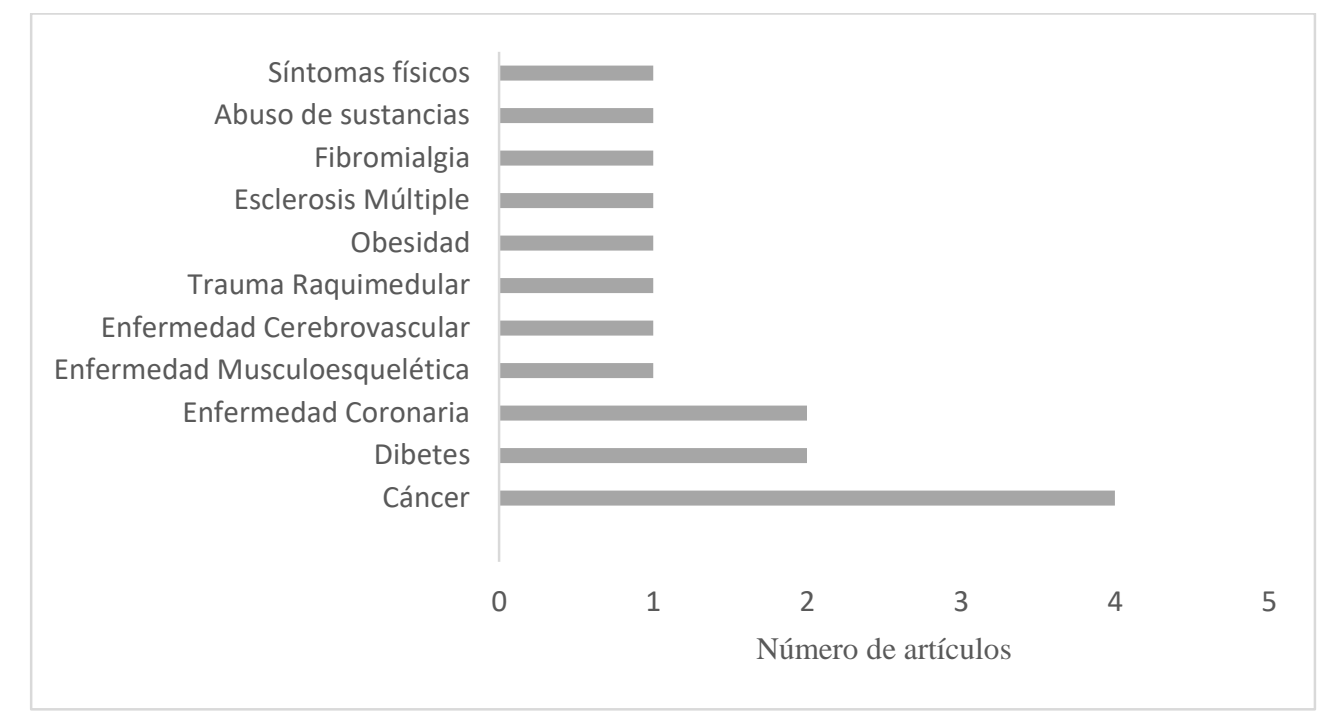

Figura 3. Enfermedades o deficiencias de salud objeto de aproximación desde la psicología positiva en los artículos revisados

Bajo estas condiciones, son cada vez más comunes los abordajes bajo una perspectiva de la psicología positiva conducentes a la exploración de los mecanismos potenciales a través de los cuales, aspectos o variables positivas en el acervo psicológico de las personas pueden influir en la etiología, progreso y manejo de la condición de salud. (Aspinwall \& Tedeschi, 2010).

El diagnóstico y la vivencia del cáncer es asumido como un proceso de salud-enfermedad, a la vez que como evento traumático al cual se enfrentan los pacientes. Su abordaje desde la psicología supone entonces, una mirada no solo bajo la perspectiva clínica sino también de la psicología de la salud, conformando un área específica denominada psico-oncología (Gan et al., 2018; Casellas-Grau et al., 2014). Los estudios en pacientes con cáncer han proporcionado resultados en relación con procesos sobre sentido, resiliencia, crecimiento postraumático, (Gan et al., 2018) optimismo, sentido de coherencia, (Coyne et al., 2010), calidad de vida, bienestar y esperanza (Casellas-Grau et al., 2014).

De acuerdo con Gan et al. (2018) si los pacientes pueden lidiar de manera exitosa con sus sentimientos y finalmente beneficiarse de la adversidad, entonces pueden alcanzar el sentido, por tanto, el dar sentido juega un papel importante en el ajuste psicológico de las personas con cáncer y puede variar en función de su resiliencia. Existen por otra parte, importantes asociaciones documentadas entre el optimismo y el sentido de coherencia con la promoción de la salud y el manejo de enfermedades como el cáncer (Aspinwall \& Tedeschi, 2010) aunque autores como Coyne et al. (2010) sugieren abrir el debate respecto de la ambiguiedad de estas relaciones y la medición objetiva de los resultados en salud física. En el mismo sentido Gorin (2010) y Casellas-Grau et al., (2014) sugieren ir más allá del consenso general de la relación 
entre aspectos positivos y cáncer, y atender a los aspectos metodológicos que soportan los resultados en torno a calidad de vida, bienestar, esperanza, búsqueda de beneficios u optimismo.

En diabetes tipo 1, Bergner et al. (2018) abordaron la adherencia al tratamiento con población adolescente, trasladando hallazgos previos en adultos según los cuales, el afecto positivo se asocia con resultados en salud, en especial si se trata de condiciones en las que los factores de comportamiento juegan un papel muy importante, tal como ocurre con la diabetes. El aumento del afecto positivo ofrece una tregua al estrés crónico mejorando la motivación, y con ello, mejor capacidad para realizar tareas específicas de monitoreo como control glucémico, conductas de afrontamiento asociadas a calidad de vida y adherencia al tratamiento. (Lord, Rumburg, \& Jaser, 2015).

Además del afecto positivo, se han considerado otras variables en el abordaje de pacientes con diabetes tipo II; en su propuesta de intervención, Celano et al. (2019) introdujeron, junto con el afecto positivo, el optimismo, la esperanza y el bienestar, lo que en su conjunto se asocia a un aumento en la motivación, confianza y autoeficacia, que a su vez inciden sobre el compromiso con comportamientos saludables, autocontrol y disminución de ambivalencia, todos estos fundamentales en el proceso de recuperación en la diabetes.

La salud cardiovascular y la prevención, más que las afecciones del corazón, han sido objeto de atención bajo una mirada de la psicología positiva. En relación con la función cardiovascular, Burg et al. (2012) han señalado que una alta variabilidad de la tasa cardíaca se relaciona con un funcionamiento más adaptativo en tanto resulta ser un correlato fisiológico de la salud física, y que esta característica, se asocia con una mejor capacidad de atención plena, de regulación de las emociones y del comportamiento. Por su parte, Labarthe et al. (2016) sugirieron la noción de salud cardiovascular positiva, a la vez que presentaron un análisis y representación del curso de la salud-enfermedad cardiovascular durante la vida de una persona, introduciendo la consideración sobre las variables positivas como un soporte importante de la calidad de vida relacionada con la salud.

Otras condiciones de salud que han sido puestas bajo el lente de la psicología positiva incluyen: enfermedad musculoesquelética (Talaei-khoei, Chen, \& Ring, 2018), enfermedad cerebrovascular (Terrill et al., 2018), trauma raquimedular o lesión medular (Nierenberg et al., 2016), esclerosis múltiple (Farber, Kern, \& Brusilovsky, 2015), fibromialgia (Finan et al., 2010), obesidad (Shinn \& Timmer, 2017), abuso de sustancias (Krentzman, 2013). Estos estudios han considerado algunas características de interés de la psicología positiva como son optimismo, resiliencia, afecto positivo, satisfacción con la vida y bienestar principalmente.

El bienestar psicológico cumple un papel amortiguador para el manejo del estrés y tiene un impacto favorable en el curso de la enfermedad y de la discapacidad. (Nierenberg et al., 2016; Farber et al., 2015). De acuerdo con Farber et al. (2015), cada vez hay más evidencia que demuestra que el bienestar psicológico tiene cualidades protectoras de la salud, de manera que, en personas con esclerosis múltiple el bienestar está relacionado con menos fatiga global o angustia relacionada con la fatiga, lo que en últimas podría afectar el desempeño de roles como el ejercicio de la maternidad. En coherencia con esto, Talaei-khoei, Chen, \& Ring (2018) estudiaron el papel protector de la satisfacción con la vida contra estilos de afrontamiento maladaptativos en personas con enfermedad musculoesquelética de miembros superiores. 
Estos autores presentaron una aproximación al dolor (su intensidad e interferencia con actividades de la vida diaria) así como a la catastrofización del dolor como mediador, a partir del papel (también mediador) de la satisfacción con la vida.

El dolor sumado a una poca satisfacción con la vida, puede interferir mayormente en las actividades de la vida diaria, en razón a una mayor catastrofización del dolor. (Talaei-khoei et al., 2018). Otro aporte, pero apoyado desde una perspectiva psicofisiológica del dolor es la Finan et al., (2010), quienes se propusieron probar la relación de los genes candidatos de los sistemas catecolinérgico y opioide con la regulación afectiva positiva durante el dolor en mujeres diagnosticadas con fibromialgia. Así, estos autores identificaron dos sistemas neurobiológicos separados, pero complementarios, que han demostrado contribuir al dolor a la vez que al procesamiento afectivo.

Lay y Hoppmann (2014) en un abordaje más general del malestar, evaluaron una serie de síntomas físicos como son dolores, problemas de alimentación, de digestión, respiratorios y baja energía general junto con el afecto positivo en parejas mayores. Estos autores encuentran que, si bien existe relación entre el afecto positivo y menos síntomas físicos, esta relación está mediada por el neuroticismo conyugal (y no individual), recordando el valor y la importancia de tener en cuenta características particulares de las personas y de sus relaciones a la hora de comprender aspectos psicológicos variables en asocio con la salud física. Para Nierenberg et al. (2016) una característica fundamental que tendrían que tener en cuenta los profesionales de la rehabilitación para promover el bienestar psicológico es el optimismo, mientras que Terrill et al., (2018) reconocen la resiliencia como fortaleza a desarrollar para promover la calidad de vida en un abordaje diádico de las secuelas en casos de enfermedad cerebrovascular.

\section{Intervenciones $y$ perspectivas futuras}

La revisión realizada por Casellas-Grau et al. (2014) reveló cinco grupos de terapias que desde la psicología positiva en cáncer de mama pueden reconocerse, a saber: aproximaciones basadas en mindfulness o atención plena, expresión de emociones positivas, intervenciones espirituales, terapia de esperanza e intervenciones de creación de significado. Estos autores resaltan un claro desarrollo en este tipo de intervenciones, aunque señalan que está pendiente una clasificación y profundización que fortalezca a la larga la psicología positiva como postura teórica.

Son diversas las intervenciones que se describen en los estudios revisados, algunas están claramente dirigidas al ajuste de comportamientos saludables. (Celano et al., 2019; Bergner et al., 2018) Celano et al (2019) reportaron resultados de un ensayo que examinó la viabilidad, la aceptabilidad y el impacto inicial de una novedosa intervención que combinaba psicología positiva y entrevista motivacional en pacientes con diabetes tipo 2, de acuerdo con estos autores una intervención de psicología positiva puede mejorar los efectos de la entrevista motivacional al promover factores como el optimismo, el apoyo percibido y la confianza, vinculados a mayores resultados en las intervenciones de comportamiento saludable.

Por su parte Bergner et al. (2018) constataron también la viabilidad y aceptabilidad de otra intervención basada en psicología positiva, usando ejercicios de afecto positivo mediante mensajes por vía telefónica con llamadas o mensajes de texto. Éste último, aunque con algunos reparos, resultó presentar una mayor favorabilidad entre los adolescentes con diabetes y sus padres. Los teléfonos móviles representan 
un mecanismo ideal en adherencia al tratamiento con población joven, y esto fue corroborado en un marco teórico orientado a la inducción del afecto positivo más que en uno basado en reforzamiento de comportamientos.

Otro grupo de intervenciones corresponde más al desarrollo de características positivas en las personas teniendo como fundamento las asociaciones entre aquellas y la mejora de condiciones de salud física. De acuerdo con Nierenberg et al. (2016), las intervenciones sobre el bienestar efectivamente han demostrado resultados a largo plazo, estudios previos han comparado resultados tras 6 años en grupos comparados para las tasas de recaída de depresión y ansiedad. Estos autores desarrollaron una terapia de bienestar basada en la teoría de Riff como un modelo estructurado, directivo y orientado a problemas basado en un modelo educativo. La terapia de bienestar es una estrategia psicoterapéutica a corto plazo que se extiende de ocho a 12 sesiones, cada una de 30 a 50 minutos de duración, que puede ocurrir semanalmente o cada dos semanas.

La revisión de Casellas-Grau et al., (2014) también deja ver que la calidad de vida es un resultado comúnmente reportado en las intervenciones basadas en atención plena (mindfulness), las cuales están dirigidas principalmente a la reducción del estrés; de igual manera, reportan que las terapias positivas en general, muestran resultados en cuanto a bienestar, crecimiento postraumático, esperanza, significado, felicidad, optimismo y satisfacción con la vida en mujeres con cáncer de mama. No obstante, llaman la atención sobre el hecho de que no todas las pacientes responden de manera favorable, lo que sugiere que estas intervenciones requieren análisis particulares a fin de establecer la efectividad en condiciones específicas. En este sentido Menezes, Kiesow, Sperb, y Hertzberg (2015) también reportan que el yoga puede ayudar a fomentar respuestas psicológicas más saludables, lo que indica su potencial como estrategia de regulación de emociones, sin embargo se requiere investigación más profunda con el fin de indagar los mecanismos de incidencia y la eficacia sobre condiciones de salud particulares.

El desarrollo de intervenciones como modelos o programas con fundamento en componentes de la Clasificación Internacional del Funcionamiento de la Discapacidad y la Salud (CIF) constituyen otra vertiente importante conducente a la recuperación de factores personales positivos que contribuyen en los procesos de participación (Farber et al., 2015). A nivel teórico, el estudio de Farber et al. (2015) demuestra la sinergia del modelo CIF (incluidos los factores personales y ambientales, y la participación) y una perspectiva de psicología positiva para el campo de la psicología de rehabilitación. Lo que apoyaría una psicología positiva de la rehabilitación que capitalice las fortalezas psicosociales tras la búsqueda de mantenimiento o mejora del bienestar más que la adaptación a la discapacidad (Nierenberg et al., 2016)

La proyección de la investigación que incorpora categorías de la psicología positiva dentro de los estudios en salud física y discapacidad apunta tanto al enriquecimiento del abordaje bajo esta perspectiva, como a la consideración permanente de las implicaciones clínicas y ajustes metodológicos. En relación con la investigación a futuro, se resalta la importancia de incorporar indicadores sobre variables ya establecidas en las condiciones estudiadas, así por ejemplo, resultará importante la exploración del estrés postraumático dentro del proceso de construcción de sentido en casos de cáncer (Gan et al., 2018), el papel de la regulación emocional en condiciones de manejo del dolor en casos de fibromialgia (Finan et al., 2010), considerar las relaciones afectivas y las diferencias culturales en el ajuste a la enfermedad y la calidad de vida durante ésta, en los diferentes momentos del curso de la vida. (Terrill et al., 2018; Lay \& Hoppmann, 2014) o 
verificar lo concerniente al aporte puntual de la variabilidad y especificidad de expresiones emocionales diversas y su relación con resiliencia en particular y, con salud y bienestar en general en un campo emergente como es la emodiversidad. (Quoidbach et al., 2014).

Teniendo en cuenta las implicaciones clínicas de varios de los estudios, serán importantes los ajustes metodológicos en diseños observacionales (Gorin, 2010) y longitudinales que respalden las intervenciones

y la observación del comportamiento de variables positivas en el curso de la enfermedad (Talaei-khoei et al., 2018). El optimismo es un candidato líder para futuras investigaciones, ya que ha sido examinado en estudios longitudinales prospectivos que evalúan la modificación del efecto y demuestran asociaciones favorables altamente consistentes con los resultados cardiovasculares, incluido un menor riesgo de enfermedad cerebrovascular. (Labarthe et al., 2016). Será también valioso, el refinamiento de aquellos aspectos que demostraron efectos significativos como presencia de cuidadores, comunicación mediante mensajes de texto o las cuestiones relacionadas con frecuencia y duración en la implementación de ejercicios particulares como son afirmaciones de afecto positivo (Bergner et al., 2018).

Finalmente, se resaltan las sugerencias de Labarthe et al. (2016) que aunque relativas a la salud cardiovascular son aplicables a otros contextos de salud: validación y estandarización de instrumentos que midan fortalezas psicológicas para la salud, establecer asociaciones entre tales fortalezas y medidas de calidad de vida relacionada con la salud o funciones biológicas en la población, estudios rigurosos que determinen eficacia, costo-efectividad y viabilidad práctica de intervención en fortalezas psicológicas para la salud, evaluar las implicaciones sociales, políticas y económicas de la salud positiva y sus efectos potencialmente transformadores en la atención médica y los sistemas de salud. A juicio de estos autores, será fundamental mantener el vínculo entre las disciplinas de salud preventiva y la psicología positiva de la salud.

\section{Discusión}

Delimitar una psicología positiva de la salud requiere sin lugar a dudas fortalecer la producción y evidencia científica en esta materia (Coyne et al., 2010), superando las limitaciones de la presente revisión exploratoria. Los debates a propósito de la implementación de intervenciones basadas en una perspectiva positiva apuntan a la cuestión sobre el sentido real de los abordajes bajo una perspectiva de la psicología positiva de la salud y de la rehabilitación, esto es, si se trata de coadyuvar para sobrellevar la enfermedad y la discapacidad o más bien, de prevenir y mejorar los resultados en salud física. Esto conduce a un asunto adicional, a propósito de la medición de dichos resultados en salud como variable dependiente en términos más objetivos que subjetivos, aspecto central de la crítica a las intervenciones positivas de la salud (Shinn \& Timmer, 2017). Sin duda el marco teórico biopsicosocial de la salud constituye el mejor de los escenarios para debatir este aspecto, con lo cual tendrían que considerarse factores más allá de la función biológica y, aún más, profundizar en la comprensión sobre la complejidad de las relaciones entre las categorías psicológicas, sociales y biológicas, en donde incluso experiencias negativas o estresantes como el diagnóstico de una enfermedad crónica o discapacidad pueden asociarse a emociones positivas o fortalezas psicológicas como variables dependientes estas y no las primeras. (Frank et al., 2010).

Por ahora cabrá resaltar el papel que juega la psicología de la salud y los desarrollos de la psicología positiva en las condiciones de salud en donde el comportamiento en general y los hábitos en particular resultan centrales en asuntos como alimentación (Shinn \& Timmer, 2017), actividad física (Celano et al., 
2019) y en comportamientos adictivos (Krentzman, 2013). En un contexto en el cual los sistemas de salud se centran en la atención de la enfermedad para intentar reparar daños, la psicología positiva de la salud representa el potencial del desarrollo de la disciplina psicológica para fines sobre la clínica preventiva como hace años lo contempló la psicología de la salud. Como lo expresan Labarthe et al. (2016) "Si las intervenciones eficaces de psicología positiva se establecen a través de la calidad de vida relacionada con la salud y se convierten en servicios preventivos clínicos reembolsables a través de mecanismos de reembolso innovadores, el efecto de tales enfoques correctivos podría mejorarse en gran medida" (p. 863).

Así pues, la intersección entre psicología de la salud con gran tradición en la disciplina en general, la psicología positiva impulsada recién en la actual centuria e incluso la psicología de la rehabilitación con un campo de acción claramente definido en la división 22 de la APA, resulta en su conjunto un campo prometedor aún con muchas cuestiones por resolver dentro de la psicología científica, pero con capacidad para afrontar el abordaje en la toda línea salud - enfermedad considerando la complejidad del fenómeno en sus dimensiones biológica, psicológica y social.

\section{Conflicto de Intereses}

Los autores declaran no presentar ninguna relación de interés comercial o personal dentro del marco de la investigación que condujo a la producción del manuscrito.

\section{Colaboraciones}

Todos los autores han contribuido intelectualmente en la elaboración del documento.

\section{Referencias}

Alex Linley, P., Joseph, S., Harrington, S., \& Wood, A. M. (2006). Positive psychology: Past, present, and (possible) future. The Journal of Positive Psychology, 1(1), 3-16. Doi: $10.1080 / 17439760500372796$

Aspinwall, L. G., \& Tedeschi, R. G. (2010). The value of positive psychology for health psychology: Progress and pitfalls in examining the relation of positive phenomena to health. Annals of Behavioral Medicine, 39(1), 4-15. Doi: 10.1007/s12160-009-9153-0

Bergner, E. M., Whittemore, R., Patel, N. J., Savin, K. L., Hamburger, E. R., \& Jaser, S. S. (2018). Participants' Experience and Engagement in Check It !: A Positive Psychology Intervention for Adolescents With Type 1 Diabetes. Translational Issues in Psychological Science, 4(3), 215-227. Doi: $10.1037 /$ tps0000161

Burg, J. M., Wolf, O. T., \& Michalak, J. (2012). Mindfulness as Self-Regulated Attention Associations with Heart Rate Variability, 71(3), 135-139. Doi: 10.1024/1421-0185/a000080

Casellas-Grau, A., Font, A., \& Vives, J. (2014). Positive psychology interventions in breast cancer . A systematic review. Psyco-Oncology, 23, 9-19. Doi: 10.1002/pon.3353

Celano, C. M., Gianangelo, T. A., Millstein, R. A., Chung, W., Wexler, D. J., Park, E. R., \& Huffman, J. C. (2019). A positive psychology - motivational interviewing intervention for patients with type 2 diabetes : Proof-of- concept trial. The International Journal of Psychiatry in Medicine, 54(2), 97114. Doi: $10.1177 / 0091217418791448$

Contreras, F., \& Esguerra, G. (2006). Psicología positiva: una nueva perspectiva en psicología. Diversitas: Perspectivas En Psicología, 2(2), 311-319. Recuerado de https://www.redalyc.org/pdf/679/67920210.pdf

Coyne, J. C., Tennen, H., \& Ranchor, A. V. (2010). Positive Psychology in Cancer Care : A Story Line 
Resistant to Evidence. Annals of Behavioral Medicine, 39, 35-42. Doi: 10.1007/s12160-010-9157-9

Ehde, D. M. (2010). Application of positive psychology to rehabilitation psychology. In R. G.; M. B. Frank; Rosenthal; Caplan (Ed.), Handbook of rehabilitation psychology (2nd ed., pp. 417-424). Washington, DC, US: American Psychological Association.

Farber, R. S., Kern, M. L., \& Brusilovsky, E. (2015). Integrating the ICF with positive psychology: Factors predicting role participation for mothers with multiple sclerosis. Rehabilitation Psychology, 60(2), 169-178. Doi: 10.1037/rep0000023

Finan, P. H., Zautra, A. J., Davis, M. C., Lemery-Chalfant, K., Covault, J., \& Tennen, H. (2010). Genetic Influences on the Dynamics of Pain and Affect in Fibromyalgia. Health Psychology, 29(2), 134142. Doi: $10.1037 / \mathrm{a} 0018647$

Frank, R., Rosenthal, M., \& Caplan, B. (Eds.). (2010). Handbook of Rehabilitation Psychology (2nd ed.). Washington, DC, US: American Psychological Association.

Gan, Y., Zheng, L., \& Wang, Y. (2018). An Extension of the Meaning Making Model Using Data From Chinese Cancer Patients : The Moderating Effect of Resilience. Psychological Trauma: Theory, Research, Practice, and Policy, 10(5), 594-601. Doi: http://dx.doi.org/10.1037/tra0000325

Gorin, S. S. (2010). Theory, Measurement, and Controversy in Positive Psychology, Health Psychology , and Cancer : Basics and Next Steps. Annals of Behavioral Medicine, 39, 43-47. Doi: 10.1007/s12160-010-9171-y

Howell, R. T., Kern, M. L., \& Lyubomirsky, S. (2007). Health benefits: Meta-analytically determining the impact of well-being on objective health outcomes. Health Psychology Review, 1(1), 83-136. Doi: 10.1080/17437190701492486

Krentzman, A. R. (2013). Review of the Application of Positive Psychology to Substance Use, Addiction, and Recovery Research. Psychology of Adictive Behaviors, 27(1), 151-165. Doi: 10.1037/a0029897

Labarthe, D. R., Kubzansky, L. D., Boehm, J. K., Lloyd-Jones, D. M., Berry, J. D., \& Seligman, M. E. P. (2016). Positive Cardiovascular Health. Journal of the American College of Cardiology, 68(8), 860867. Doi: $10.1016 /$ j.jacc.2016.03.608

Lay, J. C., \& Hoppmann, C. A. (2014). Spousal Neuroticism Moderates Everyday Problem-Wellbeing Associations in Older Couples. Health Psychology, 33(8), 803-812. Doi: 10.1037/hea0000042

Lord, J. H., Rumburg, T. M., \& Jaser, S. S. (2015). Staying positive: Positive affect as a predictor of resilience in adolescents with type 1 diabetes. Journal of Pediatric Psychology, 40, 968-977. Doi: 10.1093/jpepsy/jsv042

Lupano Perugini, M. L., \& Castro Solano, A. (2010). Psicología Positiva: Análisis desde su surgimiento. Ciencias Psicológicas, 4(1), 43-56. Recuerado de http://www.redalyc.org/articulo.oa?id=459545425005

Matarazzo, J. D. (1980). Behavioral health and behavioral medicine: frontiers for a new Health Psychology. American Psychologist, 35, 807-817.

Menezes, C. B., Kiesow, L. G., Sperb, W., \& Hertzberg, J. (2015). Yoga and Emotion Regulation : A Review of Primary Psychological Outcomes and Their Physiological Correlates. Psychology \& Neuroscience, 8(1), 82-101. Doi: 10.1037/h0100353

Miller, S. M., Sherman, A. C., \& Christensen, A. J. (2010). Introduction to special series: The great debate-evaluating the health implications of positive psychology. Annals of Behavioral Medicine, 39(1), 1-3. Doi: 10.1007/s12160-010-9173-9

Nierenberg, B., Mayersohn, G., Serpa, S., Holovatyk, A., Smith, E., \& Cooper, S. (2016). Application of Well-Being Therapy to People With Disability and Chronic Illness. Rehabilitation Psychology, 61(1), 32-43. Doi: 10.1037/rep0000060

Park, C. L. (2015). Integrating positive psychology into health-related quality of life research. Quality of Life Research, 24(7), 1645-1651. Doi: 10.1007/s11136-014-0889-z

Quoidbach, J., Gruber, J., Mikolajczak, M., Kogan, A., Kotsou, I., \& Norton, M. I. (2014). Emodiversity and the Emotional Ecosystem. Journal of Experimental Psychology: General, 143(6), 2057-2066. Doi: $10.1037 / \mathrm{a} 0038025$

Reyes-Cuervo, M. ., \& Bejarano-Celis, J. (2013). Estudio Bibliométrico de la Revista Rehabilitation 
Psychology en los años 2006 a 2012. Revista Colombiana de Rehabilitación, 12, 58-69. Doi: 10.1017/CBO9781107415324.004

Riveros, F., \& Vinaccia-Alpi, S. (2017). Relación entre variables sociodemográficas, patogénicas y salutogénicas, con la calidad de vida en estudiantes universitarios colombianos. Revista de Psicopatología y Psicología Clínica, 22(3), 229. Doi: 10.5944/rppc.vol.22.num.3.2017.16330

Schmidt, C. K., Raque-Bogdan, T. L., Piontkowski, S., \& Schaefer, K. L. (2011). Putting the positive in health psychology: A content analysis of three journals. Journal of Health Psychology, 16(4), 607620. Doi: $10.1177 / 1359105310384296$

Seligman, M. E. P. (2003). La auténtica felicidad. Barcelona, España: Vergara.

Shinn, M. M., \& Timmer, S. G. (2017). Coaching to Improve Mealtime Parenting in Treating Pediatric Obesity. Clinical Practice in Pediatric Psychology, 5(3), 232-247. Doi: 10.1037/cpp0000204

Talaei-khoei, M., Chen, N., \& Ring, D. (2018). Satisfaction With Life Moderates the Indirect Effect of Pain Intensity on Pain Interference Through Pain Catastrophizing. Journal of Consulting and Clinical Psychology, 86(3), 231-241. Doi: 10.1037/ccp0000283

Terrill, A. L., Reblin, M., MacKenzie, J. J., Cardell, B., Einerson, J., Berg, C. A., ... Richards, L. (2018). Development of a novel positive psychology-based intervention for couples post-stroke. Rehabilitation Psychology, 63(1), 43-54. Doi: 10.1037/rep0000181

Thege, B. K., Tarnoki, A. D., Tarnoki, D. L., Garami, Z., Berczi, V., Horvath, I., \& Veress, G. (2015). Is flourishing good for the heart? Relationships between positive psychology characteristics and cardiorespiratory health. Anales de Psicología, 31(1), 55-65. Doi: 10.6018/analesps.31.1.171471

Vinaccia, S., \& Quiceno, J. M. (2012). Calidad de Vida Relacionada con la Salud y Enfermedad Crónica: Estudios Colombianos. Psychologia. Avances de La Disciplina, 6(1), 123-136.

\section{Notas:}

\section{Información de autores:}

\section{María Emma Reyes Cuervo}

Psicóloga, PhD (c) en Psicología

Escuela Colombiana de Rehabilitación, Bogotá, Colombia.

Universidad de Palermo, Buenos Aires, Argentina

mariaereyes@gmail.com

https://orcid.org/0000-0003-4902-5324

\section{Stefano Vinaccia Alpi}

Psicólogo, PhD. en Psicología Clínica y Salud.

Universidad del Sinú, Montería, Colombia

vinalpi47@ hotmail.com

https://orcid.org/0000-0001-5169-0871 